A conferência que publicamos a seguir foi proferida pelo Prof. Joël Loehr, da Universidade da Borgonha, França, no âmbito do Programa de Pós-Graduação em Letras Neolatinas da Faculdade de Letras da UFRJ, no dia 17 de abril de 2009, e constitui uma síntese de comunicaçōes feitas durante vários colóquios dedicados à obra de André Malraux e de artigos publicados em diversas revistas literárias.

Joël Loehr é um especialista da obra de André Malraux, e como tal se vem apresentando em inúmeros eventos, trazendo sempre uma leitura arguta e pessoal que contribui para a renovação dos estudos malrucianos. Sua tese de doutorado, publicada pela editora Champion em 2004 e intitulada Répétitions et variations chez André Malraux, propõe-se a reler os romances do reconhecido escritor francês de maneira outra, à luz dos escritos sobre a arte, nos quais Malraux afirma uma estética profundamente antirrealista, e de suas reflexões sobre a literatura, onde mostra que "o gênio do romancista está na parte romanesca que não se pode reduzir à narrativa”.

A conferência aqui apresentada é tributária dessa leitura renovadora. Vai buscar em um texto pouco difundido de Malraux uma visão extremamente contemporânea da literatura e da participação do escritor na sociedade moderna. Ao abordar a presença de Maio de 68 na reflexão de André Malraux, estuda, no texto de Le Miroir des limbes, três objetos simbólicos que o escritor, em seu diálogo ficcional com um personagem imaginário, utiliza: o primeiro para caracterizar a luta pelas liberdades sociais, a que assistimos nos anos 60-70; o segundo para salientar a necessidade de se repensar a forma e a função da literatura naqueles tempos, superando a voz da autoridade e fazendo ecoar a voz coletiva; e o terceiro para acentuar a força e o poder da escritura, que, como uma droga, não cessa de incentivar e alimentar, em tempos de crise, a arte e o artista em sua função maior que é, como afirma o próprio Malraux no prefácio de Le Temps du mépris (1935), a de "tornar os homens conscientes da grandeza que ignoram em si mesmos". 


\section{Malraux en Mal: CRISOLOGIE DU "TEMPS DES LIMBES"}

Joël Loehr

Les événements de Mai 68 sont pour l'essentiel réfractés dans le troisième chapitre de $\mathrm{La}$ Corde et les Souris, deuxième volume du Miroir des limbes. ${ }^{*}$ Ce chapitre est daté du 6 mai 1968.

Ce jour-là, Malraux, ministre des Affaires culturelles du Général de Gaulle, s'entretient du sens des événements qui se produisent rive gauche de la Seine avec un interlocuteur fictif. Il s'appelle Max Torrès. Après la guerre d'Espagne, cet ex-communiste serait devenu spécialiste de la chimie du cerveau à Berkeley. Il arrive rue de Valois, au Palais-Royal, pourvu d'un petit cabas, d'où sortent successivement les actes d'un congrès scientifique, un soutien-gorge et une drogue inédite, présentée comme une médecine miracle pour maux d'un temps de crise. Au moment où Max Torrès semble lui suggérer de faire, avec cette mystérieuse substance, l'expérience à laquelle Michaux et Huxley s'étaient livrés avec diverses drogues hallucinogènes, Malraux commence par repousser l'offre en rétorquant: "Je n'écris plus".

L'entretien avec Torrès a pourtant bel et bien été transcrit et constitue même un chapitre entier de l'œuvre du mémorialiste. C'est à explorer ce paradoxe que l'on s'emploiera ici.

"Crise de civilisation" et rôle du "grantécrivain"

En fin de chapitre, le ministre fait droit à certaines revendications de la jeunesse soixante-huitarde, en tenant un discours au soutien-gorge que Max Torrès avait archivé dans son cabas et qu'il vient alors de déposer sur son bureau. On peut reconnaître dans ce soutien-gorge le symbole de l'oppression des femmes que Robin Morgan avait brandi à la face de l'Amérique et jeté dans "la poubelle de la liberté".

Avec cet emblème, Malraux renvoie ainsi à un certain nombre des causes d'ordre historique et sociologique qui pouvaient expliquer les événements de Mai 68. Mais, pour l'essentiel, le mémorialiste tient à distance ces deux types de perspectives, d'historien ou de sociologue, et marque leur insuffisance. Parce que Mai est à ses

* (MALRAUX, André. La Corde et les Souris, III, in Le Miroir des limbes, OEuvres complètes, t. III, "Bibliothèque de la Pléiade", Gallimard, 1996. Toutes les références de pages renverront à cette édition.) 
yeux le symptôme d'une crise beaucoup plus profonde et qui, en réalité, couvait depuis longtemps: une "crise de civilisation", une crise des "temps modernes", qu'il considère à la manière dont un ethnologue étudie les sociétés primitives.

La première raison qui explique que la civilisation occidentale soit entrée en crise au XXe siècle, c'est, selon Malraux, que ce siècle a été celui de la décolonisation. La décolonisation a en effet contraint la civilisation occidentale à prendre conscience d'elle-même, de sa différence avec d'autres civilisations, avec d'autres cultures, qui ont à nouveau émergé à la faveur de cette politique. Mais, pour la civilisation occidentale, prendre conscience d'elle-même, c'était du même coup mesurer sa relativité, et donc douter d'elle-même.

Elle n'est cependant pas entrée en crise seulement parce qu'elle a été ébranlée dans sa prétention à l'absoluité ou à l'unicité d'un modèle universel. Il y a plus grave: on est alors arrivé à la "fin d'un monde", à la fin de ce monde auquel le XIXe siècle positiviste avait, comme Renan notamment, confié l'avenir à la science. Auschwitz et Hiroshima ont ruiné la foi dans le Progrès et en la Raison. Et même: l'homme des Lumières serait mort. Selon Max Torrès, le "freudo-marxisme" l'aurait détrôné de sa position de sujet souverain, en le dépossédant de lui-même, en faisant de l'être humain le jouet d'une part de toute une série de conditionnements sociaux, économiques, et d'autre part de déterminismes d'ordre inconscient: "Quand j'étais à la Sorbonne, on s'intéressait à l'inconscient, bien sûr ! mais pour le coloniser. Maintenant, on est colonisé par lui !", ” déclare Max Torrès à Malraux.

On voit comment, par la voix de ce personnage, se retourne de la sorte en menace de complète aliénation la libération en l'homme de toutes ses pulsions que prônaient les adeptes de Reich et de Marcuse, comment les joyeuses utopies de 68 se renversent en symptômes paradoxaux d'un malaise de la civilisation et d'un nouveau mal du siècle, comment les demandes d'émancipation deviennent l'expression d'un nihilisme destructeur, d'une défaite de l'Homme et de la pensée hérités du siècle des Lumières. "Il y a quelque chose de masochiste dans cette foutue époque [...]. Tous admirent Sade", * ajoute Torrès.

Pour Malraux lui-même, le mal viendrait du fait qu'au lieu de chercher à interroger ce que l'homme est, ou à le "former", on ne s'occuperait plus que de ce qu'il subit. Au contraire de ce qui était l'objet, le dessein des Décades de Pontigny: on s'y était en effet as- 
signé pour vocation de fonder une école européenne de la sagesse et de refonder l'humanisme après le traumatisme de la première Guerre mondiale. S’il fait référence à Pontigny dans ce chapitre, c'est que les colloques de cette abbaye laïque se situent à mi-parcours entre les salons philosophiques du XVIIIe siècle, d'où Max Torrès (auquel Malraux prête significativement le visage et le rictus de Voltaire) semble issu comme un revenant, et les congrès scientifiques de la deuxième moitié du XX $X^{e}$ siècle, qui représentent, en réserve ou en puissance, la possibilité d'une nouvelle Internationale de l'Esprit.

On reviendra plus loin sur la plaquette de l'un de ces congrès que Torrès transporte dans son cabas et remet pour finir à celui qui prétend ne plus écrire. Avant de pouvoir interpréter le sens de ce geste et de l'étrange réplique par laquelle Malraux semble lui-même se destituer de sa stature d'auteur, il convient d'interroger le rôle qui pourrait être dévolu à l'écrivain dans ce "temps des limbes". Plus précisément: au "grantécrivain".

Appelons "grand écrivain" celui qui témoigne du plus haut degré possible d'implication d'une conscience dans l'histoire de son temps. Autrement dit, et pour reprendre les termes consacrés: l'écrivain "engagé". Indiscutablement, Malraux l'a été, surtout dans les années vingt et dans les années trente, au temps de la lutte contre le colonialisme et le fascisme, et notamment durant la guerre d'Espagne. La mémoire de cette époque-là, où Malraux était proche des communistes, reste gravée, inscrite, lovée au plus profond du ministre mémorialiste. En début de chapitre, Malraux se rappelle qu'il "étai $[t]$ allé parler à Berkeley pour l'Espagne" en 1938 (Il y avait tenu un discours où il appelait à rejoindre les Républicains espagnols en lutte contre les fascistes); en fin de chapitre, c'est à cet ethos du romancier combattant que, depuis Berkeley encore, on semble faire appel à nouveau, en reprenant dans un "slogan” une phrase prononcée par un personnage de L'Espoir. "Mais en 68, Malraux, officiant zélé du pouvoir gaulliste, ne peut certes retourner là-bas haranguer une foule du haut d'une tribune. Lorsque Malraux oppose une fin de non-recevoir à la requête qu'on lui lance depuis l'un des foyers les plus importants de l'agitation étudiante aux USA, lorsqu'il déclare ne plus écrire, il faut donc en un sens comprendre que, trente ans après la guerre d'Espagne et devenu ministre du Général de Gaulle, il ne saurait retrouver, restaurer en lui cet ethos de romancier combattant qu'il avait légué à son lectorat par ses romans, des Conquérants à L'Espoir. 
* (Néocritique, postface par André Malraux à Malraux, Etre et Dire Paris: Ed. Plon, 1976: 308.)
On pourrait objecter que ce n'est pas parce que Malraux a cessé d'écrire des romans “engagés" qu'il a cessé d'écrire tout court: il y a les Ecrits sur l'art, il y a surtout les Antimémoires. Allons plus avant: le ministre mémorialiste a su si bien concilier activité littéraire et rôle politique, que l'un a même soutenu l'autre jusqu'à la publication, un an avant les événements de Mai, du premier volume du Miroir des limbes (Antimémoires a été un immense succès éditorial). Non seulement c'est l'officiant du Général de Gaulle qui a écrit ce premier volume, mais c'est même fort de son statut d'émissaire spécial que le mémorialiste a pu rencontrer les grands hommes de son siècle (Nehru, Mao etc.) et qu'il a pu rapporter des entretiens (plus ou moins authentiques: c'est une autre question) qui n'ont pas peu contribué à conforter en retour son prestige, son aura et son autorité de "grand écrivain".

\section{"Mort de l'auteur" et "fin du héros"}

Que s'est-il donc passé en Mai 1968 pour qu'il renonce à sa plume et donc à son magistère? Car, en Mai 1968, Malraux ne semble plus avoir avec ce statut que la distance de la dérision et même de l'autodérision: dans les dernières pages, il donne à entendre qu'il lui reste certes des "admirateurs", mais "lointains", outreAtlantique plus exactement. "Nous faisons partie de leur exotisme", dit-il. 'Sans doute ce pronom enrôle-t-il d'abord des noms comme ceux de Voltaire et de Diderot, de Goethe ou de Hugo, bref de toute une lignée de grands écrivains. Dans un texte qui s'intitule Néocritique, publié en 1976, citant les noms de Voltaire et de Goethe, Malraux souligne que l'écrivain n'exerce pas, en Amérique, “le magistère que lui reconnut l'Europe"* et que le grand écrivain européen peut donc encore conserver là-bas, vu de là-bas, son prestige, mais précisément et seulement comme mythe.

Peut-être ce pronom, collectif indéterminé, va-t-il jusqu’à englober aussi ceux qui, en France et depuis la seconde Guerre mondiale, ont prétendu relayer les voix de Voltaire, de Diderot ou de Hugo. Peut-être comprend-il ceux qui ont occupé la place qu'avait Malraux lui-même dans les années vingt et trente, ceux qui se sont présentés à leur tour comme des veilleurs vigilants et ont représenté le plus haut degré possible d'implication d'une conscience dans l'Histoire, par quoi le grand écrivain se définit et trouve sa justification.

Mais Malraux a pu considérer que l'intervention d'Aragon boulevard Saint-Michel, le 9 mai 68, avait en réalité signé l'arrêt de 
mort, marqué la fin du rôle du "grand écrivain" dans l'Histoire: Aragon a été copieusement sifflé par les étudiants, qui ne voulaient pas de ce maitre à penser leur révolte. Dans des événements qu'il a perçus comme le symptôme d'une profonde crise de civilisation, mais qu'il a qualifiés aussi de "Saturnales", ' de grand carnaval, Malraux a vu en outre la fin du héros: l'homme exemplaire aura peut-être trouvé ses derniers avatars au moment des guerres et des révolutions du XXe siècle; mais aucune figure n'émerge d'une révolution que Raymond Aron considérait "introuvable" et où Malraux ne reconnaît ni chevalier, ni moine soldat, pas même un bolchevik.

Mêmes constats de la mort du "grand écrivain" et de la "fin du héros" chez Maurice Blanchot. Mais pour se réjouir au contraire de leur disparition. Blanchot dans l'Entretien infini déclare: "Le littérateur comme existence d'exception [...] - le héros - n'ont heureusement plus de place même dans nos mythes. Certes les vanités demeurent. Le 'Je' littéraire continue à se montrer. On parle encore des grands écrivains, des grands artistes. Personne n'y attache d'importance. Ce sont d'anciens échos qui achèvent de retentir". Et il en appelle pour sa part à accompagner et à accélérer "la fin du héros", pour que la littérature revienne "là où elle se joue" et où "il ne saurait être question d'immortalité, de puissance, ni de gloire”. *

En dépit de positions politiques et de conceptions du rapport entre littérature et vie publique diamétralement opposées, en Mai 68 et tous deux à distance de l' "engagement sartrien", Malraux et Blanchot se rejoignent cependant dans la lecture du sens des événements. ${ }^{*}$ Ces derniers ne contestent pas seulement l'autorité des Pères et celle de l'Etat; ils réfutent le grand Artiste comme ils refusent aussi tous les Maîtres à penser. Celui qui prétend exercer encore quelque magistère, a fortiori celui du "grantécrivain", est frappé de dérision; quiconque s'avance comme Auteur et pour parler du haut de son Autorité s'expose à être conspué, comme Aragon l'a été boulevard Saint-Michel.

Mais, en réalité, Malraux n’a certes pas pour autant renoncé à écrire. Quand il déclare qu'il n'écrit plus, ce qu'il faut donc bien finir par reconnaitre, ce n'est pas l'aveu d'une panne d'inspiration, ce n'est pas non plus seulement un renoncement obligé à l'ethos d'autrefois, c'est bien plutôt l'affirmation d'une écoute de ce que Mai a remis en cause et en question, et qui regarde non seulement la stature du "grand écrivain" mais aussi le statut et la fonction d' "Auteur". En d'autres termes, il faut bien poser l'hypothèse que
* (lbidem: 565.)

* (BLANCHOT, Maurice. L'Entretien infini, Paris, Gallimard, 1969: 555.)

* (Pour une confrontation entre Malraux et Blanchot au regard de Mai 68 , voir notre article "En mai, un livre sur Mai?". Poétique 156, novembre 2008: 403-419.) 
* (Néocritique, op.cit.: 297337.)
Malraux s'est lui-même destitué de la stature avec laquelle il avait signé les Antimémoires, encore renforcée par leur succès éditorial, et qu'il a pris acte de la fin de l'autorité de l'Auteur.

Il s'est ouvert aux yeux de Malraux une première possibilité de continuer à interroger l'Homme (ce qui demeure l'objet, l'objectif de sa méditation ininterrompue) dans un écrit cependant affranchi de l'autorité d'un Auteur. C'est celle que Max Torrès conservait dans son petit cabas depuis le début du chapitre et qui en sort à la fin. Malraux s'y fait remettre alors les Actes d'un congrès scientifique auquel son spécialiste de la chimie du cerveau aurait participé. Ces Actes de congrès ne contiennent pas seulement une pharmacopée de savant pour temps de crise, ils renferment aussi la forme d'un écrit qui, justement, ne soit pas le livre d'un auteur. Malraux trace les contours de sa définition dans une postface à celui qui lui a été consacré sous le titre Etre et Dire: il le nomme "Colloque". Le colloque est à ses yeux le genre moderne par excellence, parce qu'il relève d'une opération de montage entre des textes d'individus qui souvent ne se connaissent pas entre eux et dont la rencontre est plus ou moins aléatoire, et parce que les Actes d'un colloque sont donc une forme qui consacre la fin de la perspective unique et continue d'un auteur doté de toute son autorité, au bénéfice d'une parole collective, plurielle et à multiples facettes, disons avec Malraux: "cubiste". C'est aussi selon ce dernier le modèle d'une "pensée interrogative", parce qu'un colloque "remplace souvent les réponses par une dialectique des questions".

\section{Pharmakon et pharmakos}

Il est un autre modèle de ce que Malraux appelle une "dialectique des questions". Avant de pouvoir justifier qu'on nomme ici celui qui en a le premier proposé l'exemple, il faut cependant d'abord revenir à cette mystérieuse substance que Torrès apporte dans son sac à farces et attrapes.

Notons d'abord que Max Torrès, personnage agité et turbulent, surgit dans le bureau du ministre au Palais-Royal comme le bouffon fait irruption auprès du Roi Lear. Torrès semble en effet prendre ce rôle de practical joker que Shakespeare assigne à ses Fous, c'est-à-dire le rôle de celui qu'on fait rentrer en scène quand tout va mal. Car la vocation principale du practical joker est de procurer aux âmes malades un remède. 
Mais qu'est-ce donc exactement que cette médecine-miracle que Max Torrès tend à Malraux? Un de ces neuroleptiques qui périmèrent la camisole de force? Un agent hallucinogène? Le LSD que les Beatles célébraient en 1967? On pourrait certes penser à l'une ou l'autre de ces substances. Mais ce sont des trompe l'œil. A la vérité, cette drogue qui promet "la fin des drogues", ce remède au mal extrait du mal même, a surtout les caractéristiques du pharmakon. Et d'abord parce qu'elle n'a justement pas de nom. La dernière page du chapitre fait tourner autour de cette mystérieuse substance l'énumération de toute la panoplie des psychotropes disponibles à l'époque: depuis le haschich jusqu'à l'héroïne en passant par la mescaline. Mais jamais elle ne la désigne autrement que par des périphrases, et elle laisse finalement vacante la place de son nom. Si elle n'a pas de nom, c'est parce qu'elle est à proprement parler le pharmakon, contradictoirement mais indissolublement remède et poison, substance beaucoup trop paradoxale par conséquent pour pouvoir se fixer dans l'identité d'un nom.

Malraux en souligne de multiples manières le caractère foncièrement paradoxal: elle éveillerait non pas la vigilance du Contrôle des stupéfiants, mais sa jalousie; c'est une drogue, mais qui n'entraînerait aucun des effets par lesquels une drogue se définit; elle vous ouvrirait les portes du paradis, même, et peut-être surtout, si vous êtes athée; elle ne provoque pas un état second mais ramène au contraire à un énigmatique "état premier", et qui s'apparente en même temps à la mort.

On comprend l'embarras de Torrès, chargé par le collègue qui l'a découverte de remettre à Malraux cette stupéfiante substance: le don ne peut en être lui-même que paradoxal, tout à la fois salutaire et létal. Mais s'il est venu trouver Malraux à Paris, c'est qu'il n'est pas de pharmakon sans pharmakos qui en absorbe, en incorpore et en incarne les vertus, dans le rôle sacrificiel d'un missionnaire émissaire.

Reste à savoir pourquoi il faut que Torrès aille chercher ce jourlà un pharmakos de l'autre côté de l'Atlantique. Revenons d'abord sur le choix de la date du 6 mai, qui n'était pas la journée d'émeutes la plus significative. Malraux aurait pu en choisir bien d'autres. Malraux a choisi cette date moins en réalité parce qu'elle est dans le calendrier des historiens celle de la deuxième journée d'émeutes à Paris, que parce qu'elle correspond, dans le calendrier grec, à la fin des Thargélies, fête rituelle au cours de laquelle on sélectionne une victime à sacrifier pour conjurer maux et fléaux qui menacent 
* (Blanchot ouvre le chapitre intitulé "Vaste comme la nuit" sur le souvenir d'une lettre de Kafka à Brod, et montre que l'écrivain "se livre radicalement au mal". BLANCHOT. L'Entretien infini. op.cit: 465 .) et frappent la cité, et parce qu'on ne peut trouver une issue à un temps de crise que par un geste sacrificiel.

Pour trouver des exemples d'écrivains qui aient eu en personne le sens du geste sacrificiel, jetant un pont par-dessus l'Atlantique, Malraux commence par se tourner vers l'expérience à la fois poétique et psychédélique d'un Michaux et d'un Huxley. Sans doute ces deux noms apparaissent-ils en cohérence avec des pages qui parlent de mescaline et d'opiacés. Mais, plus profondément, ces deux écrivains interviennent dans le chapitre parce qu'ils sont exemplaires de la vocation de l'écrivain moderne. Celle-ci n'est plus de s'impliquer en conscience dans l'Histoire de son temps, comme elle a pu l'être aux siècles de Voltaire et de Hugo, et encore dans la première moitié du XXe; elle n'est plus de se dresser de toute sa stature sur le devant de la scène, et d'y parler en son nom propre, depuis une estrade ou un tonneau.

La mission de l'écrivain moderne répond à celle que s'était assignée le poète des limbes, et que Michaux et Huxley relaient par leurs aliénations expérimentales: non plus être l'homme de l'Universel, comme l'était encore Hugo, mais être, comme Baudelaire, celui qui sonde les abîmes de l'Anonyme. La mission de l'écrivain moderne consiste en effet à défixer son identité, pour la dissoudre dans l'anonymat de maux qui ne soient plus les siens, mais ceux de tous les hommes de son temps.

"Bouc émissaire de l'humanité" (disait Kafka), il a pour tâche d'approfondir le mal de son siècle, en descendant dans les limbes comme on descend aux enfers, pour en rapporter l'expérience, et pour la communiquer aux hommes. Il va jusqu'au fond du malaise et de l'informe, parce que l'espace et le temps des limbes sont aussi un monde d'attente et de latence, qui recèle des virtualités et des potentialités, comme le montre la section intitulée "Lazare" dans Le Miroir des limbes, où Malraux, hospitalisé, frôle la mort de près.

Le mythe lazaréen d'une plongée dans les limbes est le symétrique de ce qu'est, chez Blanchot, le mythe d'Orphée. Dans la dernière partie de L'Entretien infini, c'est à la poésie, que Blanchot confie la vocation d'une descente aux enfers. Au milieu du Miroir des limbes, Malraux lui se détourne finalement des expériences poétiques auxquelles Torrès semblait lui suggérer de se livrer, et il n'est pas encore prêt non plus à sombrer dans le sommeil de Lazare, sur quoi s'achèvera l'entreprise mémorialiste. 
Il y a en tout cas une objection majeure à ce que Malraux fasse avec sa drogue l'expérience que Michaux et Huxley ont faite avec la mescaline: l'objection, c'est précisément qu'il n'écrit plus. Cependant, si Malraux n'écrit plus, c'est bien parce qu'il ne le veut pas: ce n'est pas un aveu d'impotence littéraire, c'est un choix, celui d'abdiquer son propre statut et sa propre stature, en un temps où le prestige du grand écrivain comme l'autorité de l'Auteur sont disqualifiés.

Ainsi Malraux finit-il bien en effet par s'établir dans le rôle de “celui qui n'écrit pas", continuant néanmoins à parler et à questionner en parlant. Mais celui qui n'écrit pas, qui n'est pas un Auteur et ne veut pas de son autorité, celui qui parle néanmoins et qui questionne toujours, porte un nom: on aura reconnu une référence à Socrate. Et ce n'est certes pas un hasard si, dans un chapitre rigoureusement composé, Malraux fait apparaitre son nom en son épicentre. On comprend mieux aussi pourquoi (après avoir, comme le montrent les avant-textes du chapitre, beaucoup hésité sur la date), Malraux s'est finalement arrêté sur celle du 6 mai: cette date, à la vérité, consigne moins telle journée d'émeutes qu'elle ne commémore le jour de la naissance du philosophe, à la fin de la première semaine du mois des Thargélies. Car l'éveilleur de conscience n'est né que parce qu'il avait pour destin d'endosser le rôle sacrificiel du pharmakos, en avalant la ciguë. Et c'est donc avec le ton injonctif de Socrate que Malraux commande à Torrès de lui donner les sachets du pharmakon, indissolublement poison et remède.

Après avoir remis au mémorialiste ce qu'il devait lui remettre, Max Torrès gagne la porte avec son petit cabas. Malraux semble lui s'apprêter à un geste émissaire et suicidaire, aussi altruiste que celui du philosophe, parce que c'est sa mission de le faire, comme Socrate n'avait d'autre destin que d'incorporer le pharmakon. Sauf peut-être à considérer que, elle-même pharmakon, poison, remède, "l'écriture aussi est une puissante drogue [et que] les valises sont pleines de pages blanches qui veulent être écrites", comme il le dira au Général de Gaulle.”

\section{Resumo}

O autor fala sobre a presença de Maio de 68 na reflexão de André Malraux que, simbolicamente, aborda a luta pelas liberdades sociais, a superioridade do gênero "colóquio" em função da diversidade de vozes e em detrimento da voz autoral, e, por fim, o poder da escrita e a missão do escritor em tempos de crise.

* (MALRAuX, André. La Corde et les Souris, IV, op. cit.: 598.)

Palavras-chave: André Malraux; Le Miroir des limbes; Maio de 68; crise da civilização; morte do autor. 


\begin{abstract}
Key words: André Malraux; Le Miroir des limbes; May68; Civilization's crisis; death of the author.
\end{abstract}

Mots-clés: André Malraux; Le Miroir des limbes; Mai68; crise de civilisation; mort de l'auteur.

\section{Abstract}

The author addresses the presence of May-68 in André Malraux's thinking, which tackles symbolically the fight for social liberties, the advantage of the genre of "colloquium" due to its multiple voices to the detriment of the authorial voice, finally discussing the power of writing and the writer's mission in a moment of crisis.

\section{Résumé}

L'auteur parle de la présence de Mai-68 dans la réflexion d'André Malraux qui, symboliquement, aborde la lutte pour les libertés sociales, la supériorité du genre "colloque" en fonction de la diversité des voix et au détriment de la voix auctorale, et, enfin, du pouvoir de l'écriture et de la mission de l'écrivain dans les temps de crise. 